\title{
Metacognition in animals: Trends and challenges
}

\author{
Jonathon D. Crystal \& Allison L. Foote \\ University of Georgia
}

\begin{abstract}
There is widespread agreement that metacognition is not demonstrated if alternative explanations account for putative metacognition data. However, there is less agreement on which studies are protected from alternative explanations. We have argued that existing experiments on uncertainty monitoring can be explained by low-level explanations without assuming metacognition (Crystal \& Foote, 2009). The field would benefit from the development of accepted standards for what is required to produce a convincing example of metacognition in animals.
\end{abstract}

Keywords: Metacognition, comparative metacognition, uncertainty monitoring, metamemory, quantitative modeling.

\section{Introduction}

The collection of articles on metacognition in Comparative Cognition \& Behavior Reviews provides an opportunity to look back at previous trends and look forward to future research and challenges for the future.

\section{Trends}

Each contribution (Crystal \& Foote, 2009; Hampton, 2009; Jozefowiez, Staddon, \& Cerutti, 2009; Smith, Beran, Couchman, Coutinho, \& Boomer, 2009) to the series on metacognition recognized the burden of ruling out alternative explanations before concluding that a genuine metacognitive process is responsible for data. Hampton pointed out that "...there is a high bar to clear in terms of ruling out alternative mechanisms for metacognition before we can conclude that any nonhuman animals engage in private metacognition" (ms. p. 22) and "we can only infer private metacognition in nonhumans by excluding likely public mechanisms" (ms. p. 7). Smith et al. noted that "even when animals perform in a way that might demonstrate metacognition, researchers should

Jonathon D. Crystal and Allison L. Foote, Department of Psychology, University of Georgia, Athens GA 30602-3013

This work was supported by National Institute of Mental Health grant R01MH080052 to JDC. The content is solely the responsibility of the authors and does not necessarily represent the official views of the National Institute of Mental Health or the National Institutes of Health. Correspondence concerning this article should be addressed to JDC at jcrystal@uga.edu. consider carefully the alternative possibility that these performances can be explained through low-level, associative mechanisms based in stimulus cues and reinforcement contingencies. Morgan's Canon has seldom had a fatter target to shoot at than animal metacognition" (ms. p. 4). Jozefowiez et al. stated that “...BEM, which lacks any metacognitive ability-lacks anything beyond basic discrimination processes-satisfies the two generally accepted criteria for metacognition" (ms. p. 5), and "BEM is also able to account for data showing that animals are able to generalize the use of the uncertain response to new tasks" (ms. p. 6). Crystal and Foote noted that "if the principles of associative learning or habit formation operating on a primary representation may account for putative metacognitive data, then it would be inappropriate to explain such data based on metacognition (i.e., based on a secondary representation); the burden of proof favors primary representations, by application of Morgan's canon" (ms. pp. 4-5). Overall, there is widespread agreement that the burden of proof, in this domain, is on providing evidence that uniquely implicates a genuine example of metacognition (i.e., private metacognition, secondary representation), and until such evidence is provided, the cautious interpretation is to note that lower-level explanations are sufficient to explain the data.

However, the contributions differed in the assessment of the status of empirical evidence with respect to ruling out alternative explanations. Hampton (2009) and Crystal and Foote (2009) noted that the bar has not been reached in many studies (they differed somewhat on the prospects of reach- 
ing this bar with existing methods). Jozefowiez et al. (2009) pointed out that although their model does not explain all aspects of the data that they reviewed, other proposals may explain it without needing to use the concept of metacognition. Smith et al. (2009) noted that the bar has not been met in several studies but concluded that the bar has been met with a selection of recent approaches; they reached this conclusion by determining that some tasks are sophisticated and therefore do not call for low-level explanations. Each article in this series noted that it is not necessary to assume uncertainty monitoring in order to produce apparently functional use of the uncertainty response in at least some experiments. There was less agreement on which studies are protected from low-level, alternative explanations.

Tests of a stimulus-response hypothesis were well integrated into the examination of alternative hypotheses. However, tests of a stimulus-independent hypothesis (i.e., the implications of a low, flat threshold with constant attractiveness across stimulus conditions, as proposed by Smith, Beran, Couchman and Coutinho, 2008), needs to be added to the analysis of metacognition. Although Smith et al. (2009) pointed out that a signal detection account of metacognition (e.g., Smith et al., 2008; Smith, Shields, \& Washburn, 2003) may correspond to high-level and low-level versions, the response-strength stimulus-independent account (Smith et al., 2008) was offered specifically as a low-level alternative to metacognition (i.e., it does not have a high-level version). As we argued (Crystal \& Foote, 2009), although a transfer test is an adequate test of a stimulus-response hypothesis, transfer tests do not appear to be an adequate test of the stimulus-independent hypothesis. Moreover, the operation of residual reinforcement variables (as we documented in Crystal \& Foote, 2009) further complicates the ability to rule out low-level explanations. In Hampton's (2009) terminology, generalization to new environments is not adequately ruled out by transfer tests, in our view, because an association with environmental cues is not needed to produce a low-level, alternative explanation of adaptive control of behavior based on public information.

\section{Challenges}

It is striking that there does not appear to be an accepted standard for what it would take to produce a convincing example of metacognition in animals. We like Hampton's (2009) Table 1, which provides a systematic, thorough, and logical approach to analyzing candidate explanations and evaluating if each has been ruled out. There may be other explanations (as Hampton acknowledges and Jozefowiez et al. (2009) point out), but these explanations should be proposed and the systematic analysis extended. As Hampton points out, it will only be by ruling out alternatives that metacognition can be convincingly claimed.
Our view is that the systematic analysis (and the development of convincing evidence) will be accelerated by a complete application of low-level explanations to existing methods. If we do not recognize the limitations of existing methods, then we will undercut the motivation to develop new approaches that might have the potential to provide a compelling case for metacognition. It is a big challenge, but concluding that we have already accomplished it (if we have not) is a barrier to overcoming the challenge.

The challenge for the future is to develop new techniques that document the use of a secondary representation (in our terminology; Crystal \& Foote, 2009) or private metacognition (in Hampton's 2009 terminology). Importantly, such a new method would need to produce data that is not predicted by application of a primary representation, given the array of existing stimulus-response and stimulus-independent hypotheses.

\section{References}

Crystal, J. D., \& Foote, A. L. (2009). Metacognition in animals. Comparative Cognition \& Behavior Reviews, 4, 116.

Hampton, R. R. (2009). Multiple demonstrations of metacognition in nonhumans: Converging evidence or multiple mechanisms? Comparative Cognition \& Behavior Reviews, 4, 17-28.

Jozefowiez, J., Staddon, J. E. R., \& Cerutti, D., T. (2009). Metacognition in animals: How do we know that they know? Comparative Cognition \& Behavior Reviews, 4, 29-39.

Smith, J. D., Beran, M. J., Couchman, J. J., \& Coutinho, M. V. C. (2008). The comparative study of metacognition: Sharper paradigms, safer inferences. Psychonomic Bulletin \& Review, 15, 679-691. doi:10.3758/PBR.15.4.679

Smith, J. D., Beran, M. J., Couchman, J. J., Coutinho, M. V. C., \& Boomer, J. B. (2009). Animal metacognition: Problems and prospects. Comparative Cognition \& Behavior Reviews, 4, 40-53.

Smith, J. D., Shields, W. E., \& Washburn, D. A. (2003). The comparative psychology of uncertainty monitoring and metacognition. Behavioral and Brain Sciences, 26, 317 $373 . \quad$ doi: $10.1017 / \mathrm{S} 0140525 \mathrm{X} 03000086$ 